\title{
Outburst Properties of V1504 Cyg and V344 Lyr
}

\author{
J. K. Cannizzo ${ }^{1,2}$ \\ ${ }^{1}$ CRESST, Dept. of Phys., UMBC, Baltimore, MD 21250, USA \\ ${ }^{2}$ NASA/GSFC/Astroparticle Physics Lab., Greenbelt, MD 207r1, USA \\ Corresponding author: John.K.Cannizzo@nasa.gov
}

\begin{abstract}
I begin by reviewing dwarf novae and the disk instability theory, and then present an overview of three ideas for producing superoutbursts in the SU UMa stars - the thermal tidal instability, irradiation-induced secondary mass overflow, and the plain vanilla disk limit cycle instability. I discuss the properties of the outbursts in two SU UMa systems observed by Kepler in the context of the three theories. I conclude with a look beyond the SU UMa systems.
\end{abstract}

Keywords: cataclysmic variables - dwarf novae - optical - photometry - - individual: V1504 Cyg, V344 Lyr, U Gem, SS Cyg.

\section{Introduction}

\subsection{Disk instability model}

The outbursts in dwarf novae (DNe), a subclass of cataclysmic variables (CVs - Warner 1995), are thought to be due to a limit cycle instability operating within the accretion disk (Smak 1984) that episodically stores up matter (during quiescence) and dumps a fraction of it onto the accreting WD (during outburst). The basic disk instability model is now generally accepted as the correct explanation for dwarf nova outbursts (Smak 1984, Cannizzo 1993a, Lasota 2001). The strongest point of the model is its prediction of a dividing line between novalike and dwarf nova systems (Smak 1984), a line which has no free parameters ${ }^{1}$. For the systems above this line, the rate of accretion feeding into the outer disk is such that the entire disk is always in a hot, ionized state. Other strengths of the theory include its natural ability to account for slow and fast decays of DN outbursts as being due either to viscous or thermal decay, the natural tendency of the model to produce alternating long and short outbursts (Cannizzo 1993b), and the fact that the outer disk radius $r_{\text {outer }}$ continues to contract in quiescence following a DN outburst, whereas in the secondary mass-overflow model there is a one-to-one relation between disk luminosity and $r_{\text {outer }}$ (Ichikawa \& Osaki 1992).

Smak (1984) was the first to use the relation between the fast rate of decay in dwarf nova outbursts and orbital period (i.e., the Bailey relation) to constrain the value of the Shakura-Sunyaev (1973) alpha parameter in the ionized disk, $\alpha_{\text {hot }} \simeq 0.1$. The high fidelity $K e$ pler data now enable us to trace deviations from strictly exponential decay for the fast decays (Cannizzo et al. $2012=\mathrm{C} 12$ ), and also to utilize the slow rate of decay, wherein the entire disk resides in a hot, ionized state. The fact that the viscous and thermal time scales differ by a factor $h / r$ means that this ratio can be directly calculated to be $0.01-0.02$ (Cannizzo 1998; Menou, Hameury, \& Stehle 1999).

Although successful as a model for outbursts, it has been known for some time that there must be various add-ons to the theory in order to have a complete theory for the accretion disk, including something like a coronal siphon flow (Meyer \& Meyer-Hofmeister 1994) or some other type of evaporative instability (e.g., Shaviv \& Wehrse 1986) to transfer matter from the disk onto the WD during quiescence to get accretion.

\subsection{SU UMa systems}

The SU UMa stars represent short orbital period dwarf novae $(\mathrm{DNe}), P_{\text {orb }}<2 \mathrm{hr}$, displaying normal outbursts (NOs) and superoutbursts (SOs) - long outbursts with superhumps (SHs). Although the contrast between NOs and SOs seems quite dramatic, van Paradijs (1983) has shown that the properties of SOs, seen in systems below the period gap, can be understood as a natural continuation of the long outbursts in DNe above the period gap $P_{\text {orb }}>3 \mathrm{hr}$. In addition, SOs are defined by the existence of SHs, modulations in the outburst

${ }^{1}$ For $\sim 10$ yr it appeared that the best studied dwarf nova, SS Cyg, was on the wrong side of the line (Harrison et al. 1999, Schreiber, Hameury, \& Lasota 2003). However, this prognosis was based on an incorrect distance (159 \pm 12 pc). The corrected distance (114 \pm 2 pc) reconciles data and theory (Miller-Jones et al. 2013). 
light curve at periods a few percent longer than the orbital period. The prevailing theory is that when the outer disk edge surpasses the point of $3: 1$ resonance with the binary orbit, an eccentric disk develops that precesses progradely in the binary frame (Whitehurst 1988). More recent work indicates that the disk is not statically eccentric but has a fundamental "breathing" mode - oscillating between circular and eccentric (Montgomery 2012ab). These SHs are referred to as positive superhumps (pSHs) since their periods exceed $P_{\text {orb }}$; there also exist negative superhumps (nSHs) with periods a little less than $P_{\text {orb }}$ thought to be indicative of a tilted, retrogradely precessing disk (Patterson et al. 1993). The two disk states can and sometimes do coexist (Montgomery 2012ab).

In recent years three scenarios have emerged to account for SOs: (i) the thermal-tidal instability (TTI Osaki 1989) which combines the normal disk instability model and the precessing disk model, (ii) irradiationinduced enhanced mass overflow from the mass-losing star, and (iii) the plain disk instability (pDI) model (Cannizzo et al. $2010=\mathrm{C} 10$ ). In a recent series of papers Osaki \& Kato (2013abc) have discussed the relative merits of these three models within the context of recent Kepler observations of V1504 Cyg and V344 Lyr.

\section{Kepler Observations of V1504 Cyg and V344 Lyr}

C12 find that the durations of the NOs within a supercycle increase monotonically, whereas the durations of the quiescence intervals between NOs can either increase monotonically, or else increase to a local maximum about half way to the next SO, and then decrease. These supercycles for which the recurrence times for NOs reach a maximum roughly half way between SOs present a challenge for the TTI model, in which one expects a monotonic increase in disk mass, angular momentum, and triggering radius as the next SO approaches. Osaki \& Kato (2013c) acknowledge this difficulty, and also point out that this criticism, given by C12, applies equally well to the pDI calculations presented by C10. This is a good point - in fact the calculations presented in C10 did not show any variation in recurrence time for NOs. Figure 1 shows the latest pDI calculations of SOs in a SU UMa.

OK13a discuss the amplitude of the precursor dip and note that, at shorter wavelengths, the dip tends to stand out more strongly. One of the best examples is examples is in OY Car $\left(P_{\text {orb }}=1.51 \mathrm{hr}\right.$; Mauche \& Raymond 2000) where simultaneous visual and EUV ( $\sim 100 \AA)$ observations are presented of a SO. Embedded precursors which are simultaneously small in the optical and large in the EUV provide a challenge to both TTI and pDI models. In the TTI model, the am- plitude of the precursor can be precisely controlled by tweaking the strength of the increase in tidal torque accompanying the crossing of the $3: 1$ resonance radius by $r_{\text {outer }}$, and the delay time $t_{\mathrm{TTI}}$, delay accompanying its onset. For the SOs there can be no precursors, as in the early TTI models (e.g., Ichikawa, Hirose, \& Osaki 1993), or there can be precursors which are well-defined both in $\mathrm{V}$ and EUV wavelengths, e.g., in models with $t_{\text {TTI, delay }}=1.75 \mathrm{~d}$ (Schreiber, et al. 2004, see their Fig. 3).

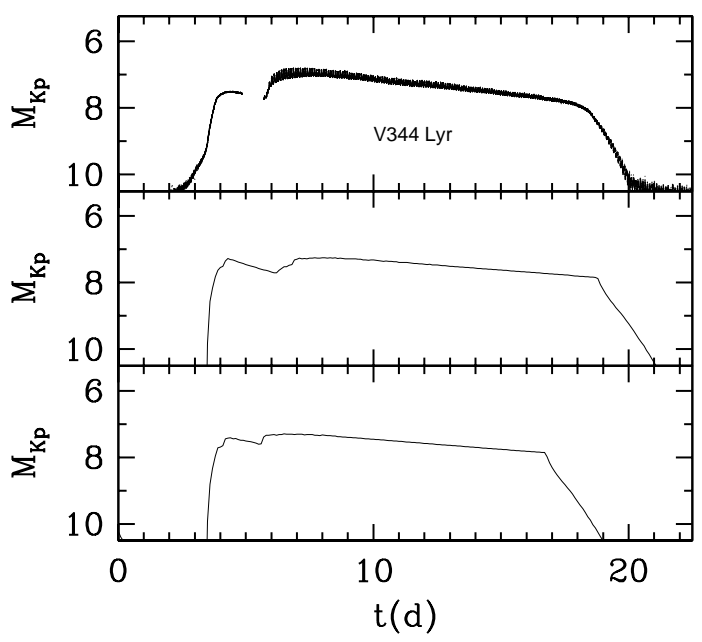

Figure 1: A comparison of Kepler data on V344 Cyg with two SOs in a long light curve using the pDI model.

A new idea introduced in OK13a is that the presence of time-varying nSHs, when they are present, gives us a handle on variations in the outer disk radius. Larwood (1998) gives a general expression for the (nodal) precessional frequency associated with a tilted disk $\omega_{\mathrm{nPR}}=\omega_{\text {orb }}-\omega_{\mathrm{nSH}}$ which is based on the surface density distribution $\Sigma(r)$ and accretion disk tilt angle $\theta$,

$$
\omega_{\mathrm{nPR}}=2 \pi \nu_{\mathrm{nPR}}=-\frac{3}{4} \frac{G M_{2}}{a^{3}} \frac{\int \Sigma r^{3} d r}{\int \Sigma \Omega r^{3} d r} \cos \theta,
$$

where $M_{2}$ is the secondary mass and $a$ the binary separation. For a nominal power law distribution in surface density,

$$
\frac{\nu_{\mathrm{nPR}}}{\nu_{\mathrm{orb}}}=-\eta \frac{3}{7} \frac{q}{\sqrt{1+q}}\left(\frac{r_{\text {outer }}}{a}\right)^{3 / 2} \cos \theta,
$$

where $\eta$ is a constant of order unity. The observed $\nu_{\mathrm{nSH}}(t)$ variations in V1504 Cyg reveal a disk contraction between NOs, superposed on a longer term expansion between SOs. This approach was criticized by Smak (2013) who argued that, if one takes the time derivative of Larwood's $P_{\mathrm{nSH}}$ equation, one obtains an expression with five terms (see his eqn. [7]), only one of which is related to the outer disk radius. 
The disk instability model provides a definitive prediction of $\Sigma(r)$ at all times, and therefore one may carry out a calculation of the long-term accretion disk evolution relevant for an SU UMa system, and do a direction comparison of $\nu_{\mathrm{nSH}}$ calculated using the exact and approximate expressions. The results are shown in Figure 2. The panels show the light curve, disk mass, disk radius, and the exact and approximate calculations for $\nu_{\mathrm{nSH}}$. The approximate expression gives a very good representation of the exact formula, which vindicates the underlying idea of OK13a and supports their use of $\nu_{\mathrm{nSH}}(t)$ to track $r_{\text {outer }}(t)$.

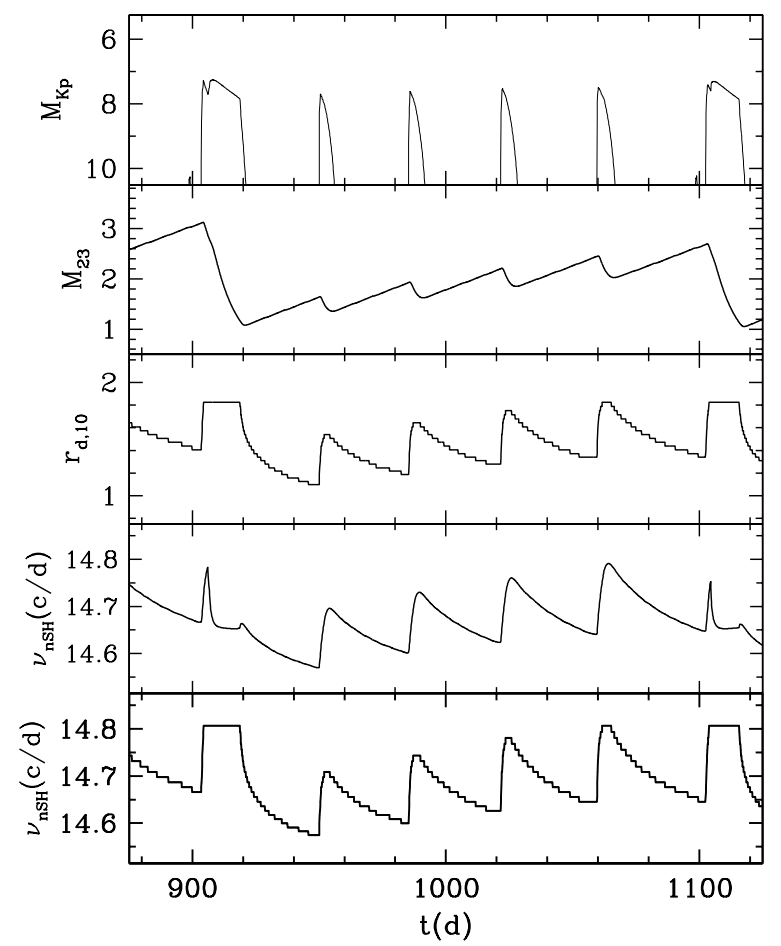

Figure 2: A calculation of the pDI model for a SU UMa system. Shown are (i) the Kepler light curve, (ii) the disk mass, in units of $10^{23} \mathrm{~g}$, (iii) the outer disk radius, in units of $10^{10} \mathrm{~cm}$, (iv) the $\mathrm{nSH}$ frequency calculated using the Larwood formula, and (v) the nSH frequency calculated using the approximate formula given OK13abc, taking $\eta=1.5$.

There is actually only one small difference between Osaki's TTI and the pDI model for superoutbursts, namely, in the TTI model there is a strong and brief contraction of the outer disk radius due to the (hypothesized) strong increase in tidal torque from the secondary star. An obvious way to distinguish between the two models is to closely monitor the outer disk radius in an eclipsing SU UMa star during the early onset of a SO to see if there is a sudden, brief decrease in $r_{\text {outer }}$. Smak (2013) argues that this observation has already been carried out and shows no such contraction; OK13c counter that the time period in question was well into the $\mathrm{SO}$ and therefore missed the start of the SO.

\section{Looking Beyond the SU UMa Systems}

OK13abc argue that the precursor in SOs is due to the onset of the TTI, whereas I have argued it is a natural consequence of the pDI. An obvious next step would be to look in detail at long DN outbursts in systems where we do not expect the TTI to operate - systems above the period gap where the outer disk radius never reaches the 3:1 radius. Even if one accepts the fundamental tenet of the TTI, namely a causal relation between Whitehurst's precessing eccentric disk and the DI, it could not possibly be a factor because the disks in long period systems are never subject to the $3: 1$ instability. If one sees precursors in long DN outbursts in these systems, then they must be due to a more general aspect of the DI.

The two best studied DNe are both longward of the period gap and should not experience the 3:1 instability - U Gem and SS Cyg. They have > $100 \mathrm{yr}$ light curves, but unfortunately the bulk of the data is not precise enough to enable any statement to be made about the detailed shape of the outburst onset. Cannizzo (2012) examined the AAVSO data for these systems, and found brief stretches of the light curves for which the number of observations entering into daily means exceeds $\sim 10^{3}$, concomitant with the use of digital photometry. Although still not on a par with Kepler data, these data do afford a more detailed look at the outburst light curve shapes than the usual data. There are two long outbursts in U Gem and one in SS Cyg for which a statement can be made concerning the shape of the outburst light curve during outburst onset; one of the U Gem outbursts shows no obvious precursor, one does, and the one SS Cyg long outburst also does. In fact, the flattened shape of the precursor is similar to that seen in Fig. 13 (panel 2) of Cannizzo (1993b) in modeling SS Cyg. These outbursts provide a hint that embedded precursors during long outbursts may not be confined to the SU UMa systems.

\section{Summary}

Kepler light curves of short period DNe have resparked interest in the nature of SOs and led to the question: Is the thermal-tidal instability needed, or can the plain vanilla version of the accretion disk limit cycle do the job all by itself? A detailed time-resolved study of an eclipsing SU UMa system during SO onset should settle the question - if there is a dramatic contraction of the disk at SO onset, the TTI would be preferred; if not, the plain DI model would be sufficient. Also, recent work 
by Osaki \& Kato has shown that the time varying $\mathrm{nSH}$ frequencies $\nu_{\mathrm{nSH}}(t)$ can be taken as a surrogate for the outer disk radius variations $r_{\text {outer }}(t)$. Finally, it may be necessary to look beyond the short period dwarf novae to gain perspective on the nature of embedded precursors in long outbursts.

\section{References}

[1] Cannizzo, J.K.: 1993a, in Accretion Disks in Compact Stellar Systems ed., J.C. Wheeler (Singapore: World Scientific), 6.

[2] Cannizzo, J.K.: 1993b, ApJ, 419, 318. doi:10.1086/173486

[3] Cannizzo, J.K.: 1998, ApJ, 494, 366. doi:10.1086/305210

[4] Cannizzo, J.K.: 2012, ApJ, 757, 174. doi:10.1088/0004-637X/757/2/174

[5] Cannizzo, J.K., Smale, A.P., Wood, M.A., et al.: 2012, ApJ, 747, 117. doi:10.1088/0004-637X/747/2/117

[6] Cannizzo, J.K., Still, M.D., Howell, et al.: 2010, ApJ, 725, 1393. doi:10.1088/0004-637X/725/2/1393

[7] Harrison, T.E., McNamara, B.J., Szkody, P., et al.: 1999, ApJ, 515, L93. doi:10.1086/311982

[8] Ichikawa, S., Hirose, H., Osaki, Y.: 1993, PASJ, $45,243$.

[9] Ichikawa, S., Osaki, Y.: 1992, PASJ, 44, 15.

[10] Larwood, J.: 1998, MNRAS, 299, L32. doi:10.1046/j.1365-8711.1998.01978.x

[11] Lasota, J.-P.: 2001, New Astron. Rev., 45, 449. doi:10.1016/S1387-6473(01)00112-9

[12] Mauche, C.W., Raymond, J.C.: 2000, ApJ, 541, 924. doi:10.1086/309489

[13] Menou, K., Hameury, J.-M., Stehle, R.: 1999, MNRAS, 305, 79.

[14] Meyer, F., Meyer-Hofmeister, E.: 1994, A\&A, 288,175 .

[15] Miller-Jones, J.C.A., Sivakoff, G.R., Knigge, C., et al.: 2013, Science, 340, 950. doi:10.1126/science. 1237145

[16] Montgomery, M.M.: 2012a, ApJ, 745, L25. doi:10.1088/2041-8205/745/2/L25
[17] Montgomery, M.M.: 2012b, ApJ, 753, L27. doi:10.1088/2041-8205/753/2/L27

[18] Osaki, Y.: 1989, PASJ, 41, 1005.

[19] Osaki, Y., Kato, T.: 2013a, PASJ, 65, 50.

[20] Osaki, Y., Kato, T.: 2013b, astro-ph/1305.5877.

[21] Osaki, Y., Kato, T.: 2013c, astro-ph/1309.3722.

[22] Patterson, J., Thomas, G., Skillman, D.R., Diaz, M.: 1993, ApJS, 86, 235.

[23] Schreiber, M.R., Hameury, J.-M., Lasota, J.-P.: 2003, A\&A, 410, 239.

[24] Schreiber, M.R., Hameury, J.-M., Lasota, J.-P.: 2004, A\&A, 427, 621.

[25] Shakura, N.I., Sunyaev, R.A.: 1973, A\&A, 24, 337.

[26] Shaviv, G., Wehrse, R.: 1986, A\&A, 159, L5.

[27] Smak, J.: 1984, Acta Astron., 34, 161.

[28] Smak, J.: 2013, Acta Astron., 63, 109.

[29] van Paradijs, J.: 1983, A\&A, 125, L16.

[30] Warner, B.: 1995, Cataclysmic Variables (Cambridge: Cambridge Univ. Press).

[31] Whitehurst, R.: 1988, MNRAS, 232, 35.

\section{DISCUSSION}

MARGARETHA PRETORIUS: If there is no tidal instability how do you make superhumps?

JOHN CANNIZZO: The tidal instability is not being disputed. In both Osaki's thermal-tidal instability model and the plain disk instability model superhumps are due to a precessing eccentric disk which becomes manifest when the outer disk radius surpasses the the $3: 1$ radius. The question is then whether this has anything to do with the superoutbursts: Osaki would say yes, I would say no.

RAYMUNDO BAPTISTA: Yesterday I showed inferred $\alpha_{\text {cold }}$ values for three quiescent SU UMa stars, which show $\alpha_{\text {cold }} \sim 10^{-1} \sim \alpha_{\text {hot }}$. My interpretation is that this eliminates the possibility of the disc instability model to explain their outbursts. Could you please comment on this?

JOHN CANNIZZO: What's the temperature of that gas?

RAYMUNDO BAPTISTA: About $6000 \mathrm{~K}$. 
JOHN CANNIZZO: One of the limitations of the standard disk instability theory has always been that it's not a complete theory - certainly it doesn't work at all to describe the quiescent state. If you take the model seriously, the plots in the movie I showed indicate temperatures in quiescence of $\sim 2000-3000 \mathrm{~K}$. Also, we see significant evidence for accretion during quiescence - mainly through soft X-ray emission, which is not predicted by the DI theory since in quiescence the accretion rate would be zero. So the bottom line is that the quiescence gas you observe must be due to something extrinsic to the DI model. 